\title{
Role of a prolonged inpatient admission when evaluating children with problematic severe asthma
}

\author{
To the Editor:
}

Severe asthma continues to be a significant cause of morbidity in children. Despite optimised standard therapy, many children remain symptomatic with subsequent impact on their quality of life and increased healthcare utilisation [1]. Children with ongoing poorly controlled asthma despite maximally prescribed treatment are identified as having problematic severe asthma [2]. Those whose poor control is due to modifiable factors including poor adherence to medications, continued exposure to allergens, social issues and psychological factors have difficult asthma. Severe therapy-resistant asthma is the term used for those with persistent symptoms despite attention to the basics of asthma management [3].

In children with problematic severe asthma, differentiating difficult asthma from severe therapy-resistant asthma is vital as correction of modifiable factors in patients with difficult asthma may result in improved symptom control and limit further investigations and exposure to expensive medications with potentially significant side effects. We have demonstrated that a structured outpatient nurse-led assessment that addresses the basics of asthma management leads to long-term benefits [1, 4]. The basics include confirming the diagnosis, ensuring correct inhaler technique, monitoring adherence, minimising allergen and smoke exposure, and assessing psychosocial factors. This involves nurse-led hospital, home and school visits, and a multidisciplinary evaluation. The assessment has been described in full previously [4]. Only once these factors have been addressed are children classified as having severe therapy-resistant asthma and consideration given to further assessments and add-on therapies such as omalizumab. Despite this rigorous assessment process, a small number of cases remain perplexing and cause concern, particularly when reported symptoms are discrepant with objective measures.

We hypothesised that selected children with problematic severe asthma would benefit from elective inpatient observation, investigations and detailed multidisciplinary assessment. We undertook a detailed assessment of children with problematic severe asthma admitted electively for an intensive inpatient stay to the Royal Brompton Hospital between 2012 and 2014. Before admission, all children had undergone an outpatient assessment and home visit as described above and were discussed at the multidisciplinary difficult asthma meeting. Information from the referring hospital, school and any other services involved in the care of the child was used to develop a detailed personalised admission plan. The admission was discussed with the families in advance. During admission all children attended the hospital school (during term times), participated in a daily physiotherapy-led exercise programme, were taken on trips out to local parks and museums, were offered psychological support and all medications were supervised and directly observed. Children were reviewed by a doctor when they reported asthma symptoms and salbutamol was only given if recommended by the doctor following review. Forced expiratory volume in $1 \mathrm{~s}$ (FEV1), bronchodilator reversibility $(\mathrm{BDR})$ and exhaled nitric oxide $(\mathrm{FeNO})$ were measured twice weekly. The asthma control test (ACT) score was measured at admission and follow-up. The Wilcoxon signed rank test or Kruskal-Wallis with post hoc test was used to compare the data at different time points.

26 children with median age of 12.4 (range 6-15) years were included. 14 (53.8\%) were male and the median duration of admission was 13 (range 6-20) days. The median prescribed inhaled steroid dose was 1000 (range 400-2400) $\mu \mathrm{g} \cdot \mathrm{day}^{-1}$ of beclamethasone equivalent. During the preceding 12 months, these

@ERSpublications

Valuable information can be obtained during inpatient assessment of children with problematic severe asthma http://ow.ly/RiKc30hzNXz

Cite this article as: Nagakumar P, Gambir N, Sanghani N, et al. Role of a prolonged inpatient admission when evaluating children with problematic severe asthma. Eur Respir J 2018; 51: 1701061 [https://doi.org/ 10.1183/13993003.01061-2017]. 
children had a median 5.5 (range 0-24) unscheduled healthcare visits, 3 (range 0-10) hospital admissions and received 4.5 (range $0-20$ ) courses of oral corticosteroids.

In the group as a whole FeNO improved significantly from admission to discharge (table 1) (38 (range 103-6) ppb versus 20.7 (range 49-5) ppb; p=0.007). FEV1 improved from 92\% (range 59-116\%) at admission to $99 \%$ (range $58-122 \%$ ) predicted ( $\mathrm{p}=0.003$ ), at discharge and BDR improved from $4.7 \%$ to $3.5 \%(\mathrm{p}=0.02)$.

Following discharge, children were followed up at a median of 128 (range 43-221) days. The follow-up outpatient clinic appointment included, as per our routine practice, measurement of FEV1, BDR, FeNO, $\mathrm{ACT}$, assessment of inhaler technique by the asthma nurse and a medical review by a respiratory physician. Improvements in FEV1 and $F$ eNO were sustained at follow-up ( $\mathrm{p}=0.03, \mathrm{p}=0.002$, respectively) (table 1) emphasising the longer-term impact of an admission on asthma control. The median ACT score improved from admission to follow-up: 11.5 (range 6-22) to 18.5 (range 9-24), p=0.005.

During admission most children remained well, likely to be because of improved adherence as all therapy was directly observed or suggesting previous over-reporting of symptoms. Only two patients remained symptomatic and had persistently abnormal lung function and elevated FeNO. They were identified as having true severe therapy-resistant asthma and had a further assessment for add-on therapies; one of the patients was started on omalizumab. The information gained as part of the admission had a significant impact on management: six patients on maintenance oral steroids had their medication weaned during admission and the oral steroids were either stopped or were at a significantly lower dose at follow-up $(\mathrm{p}=0.01)$. Eight children had normal lung function, were asymptomatic and required no salbutamol during the inpatient stay despite high levels of symptom reporting. Asthma medications were weaned either at discharge or follow-up. Six patients were referred to children's social care because of concerns regarding fabricated illness or adverse home environment contributing to poor control. In most cases, these issues had been suspected previously; however, the admission was critical in helping to obtain concrete evidence to support the referral to children's social care. Six children were diagnosed with a breathing pattern disorder, which became evident during the daily physiotherapy and activity programme or when symptoms (mis-attributed to asthma) were reported. Following intensive input from the psychologist during the admission, eight children were found to have significant psychological issues. It is to be noted that some children had more than one problem identified.

The findings of this study support anecdotal reports of improvements in asthma control in children placed in residential facilities, including specialist boarding schools and salt mines [5]. The observed improvements are likely to be a result of good adherence, as well as psychosocial support and reduction in allergen exposure. To our knowledge this is the first study of its kind to provide clear objective evidence of benefit from an elective inpatient stay regarding improvements in airway inflammation, lung function, symptoms and reduction in treatment burden. Moreover, these improvements were sustained at follow-up. Although time and labour intensive, we have demonstrated that key information can be gained in these children with high levels of healthcare utilisation. Of particular note are the valuable insights and evidence obtained enabling us to take forward safeguarding concerns. Arguably, this will have the most significant impact on a child's future.

In conclusion, an admission should be considered in children with a discrepancy in the severity of reported symptoms and objective evidence of severe disease, particularly where there are safeguarding

TABLE 1 Sustained improvement in measures of asthma control

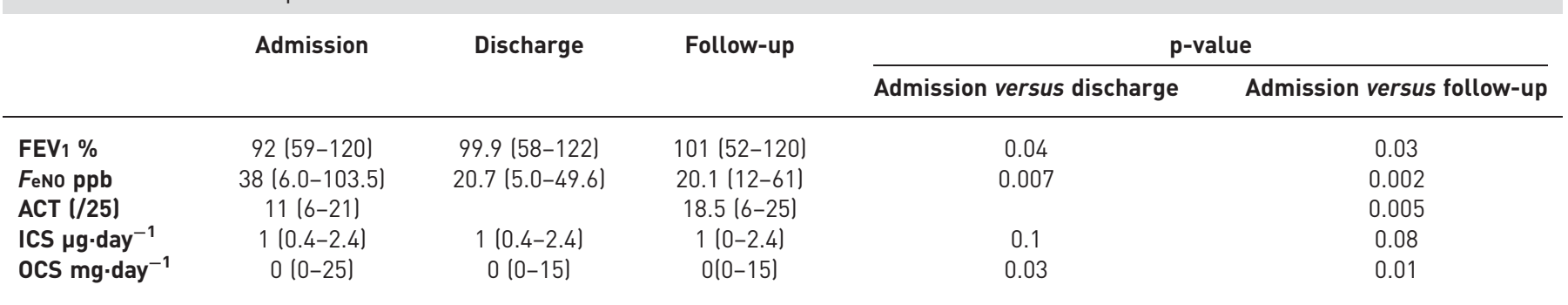

Data are shown as median and range. Groups compared by Wilcoxon signed rank test or Kruskal-Wallis test with Dunn's post hoc comparison test or Chi-squared test; significance $p<0.05$. $p$-values between admission and discharge and between admission and follow-up shown respectively. Asthma control test (ACT) scores are out of a total of 25 . FEV1: forced expiratory volume in $1 \mathrm{~s}$. FeNO: fractional exhaled nitric oxide; ICS: inhaled corticosteroids; OCS: oral corticosteroids. 
concerns. Further studies are essential to evaluate the economic impact of a prolonged hospital stay and the implications for the families.

Prasad Nagakumar ${ }^{1,2}$, Nikki Gambir ${ }^{1}$, Nina Sanghani ${ }^{1}$, Pippa Hall ${ }^{1}$, Angela Jamalzadeh ${ }^{1}$, Frances Beresford ${ }^{1}$, Sejal Saglani ${ }^{1,2}$, Andrew Bush ${ }^{1,2}$ and Louise Fleming ${ }^{1,2}$

${ }^{1}$ Dept of Respiratory Paediatrics, Royal Brompton Hospital, London, UK. ${ }^{2}$ National Heart and Lung Institute, Imperial College, London, UK.

Correspondence: Louise Fleming, National Heart and Lung Institute, Imperial College, and Royal Brompton and Harefield NHS Foundation Trust, Sydney Street, London SW3 6NP, UK. E-mail: 1.Fleming@rbht.nhs.uk

Received: June 052017 | Accepted after revision: Nov 172017

Support statement: A. Bush is an NIHR Senior Investigator who was additionally supported by the NIHR Respiratory Disease Biomedical Research Unit at the Royal Brompton and Harefield NHS Foundation Trust and Imperial College London, and PI in the Asthma UK Centre for Applied Research. S. Saglani is an NIHR Career Development Fellow. L. Fleming is an Asthma UK Senior Clinical Fellow and PI in the Asthma UK Centre for Applied Research.

Conflict of interest: Disclosures can be found alongside this article at erj.ersjournals.com

\section{References}

1 Sharples J, Gupta A, Fleming L, et al. Long-term effectiveness of a staged assessment for paediatric problematic severe asthma. Eur Respir J 2012; 40: 264-267.

2 Hedlin G, Bush A, Lødrup Carlsen K, et al. Problematic severe asthma in children, not one problem but many: a GALEN initiative. Eur Respir J 2010; 36: 196-201.

3 Bush A, Saglani S. Management of severe asthma in children. Lancet 2010; 376: 814-825.

4 Bracken M, Fleming L, Hall P, et al. The importance of nurse-led home visits in the assessment of children with problematic asthma. Arch Dis Child 2009; 94: 780-784.

5 Lazarescu H, Simionca I, Hoteteu M, et al. Speleotherapy - modern bio-medical perspectives. J Med Life 2009; 2: $76-79$. 